Musées, Patrimoine et Culture scientifiques et techniques

$131 \mid 2010$

septembre - octobre 2010

\title{
La formation professionnelle : « un enrichissement réciproque »
}

\section{Olivier Soichot}

\section{(Q) OpenEdition \\ Journals}

Édition électronique

URL : http://journals.openedition.org/ocim/121

DOI : 10.4000/ocim.121

ISSN : 2108-646X

Éditeur

OCIM

Édition imprimée

Date de publication : 1 septembre 2010

Pagination : $32-37$

ISSN : 0994-1908

Référence électronique

Olivier Soichot, «La formation professionnelle : « un enrichissement réciproque » », La Lettre de I'OCIM [En ligne], 131 | 2010, mis en ligne le 01 septembre 2012, consulté le 19 avril 2019. URL : http:// journals.openedition.org/ocim/121; DOI : 10.4000/ocim.121 


\title{
Points de vue
}

\section{La formation professionnelle : " un enrichissement réciproque »}

\author{
Depuis le mouvement de professionnalisation du secteur culturel au début des années 1980, \\ de nombreux organismes proposent des formations destinées à aider les professionnels \\ à s'adapter aux évolutions de leurs métiers. Spécialiste des questions de marketing et de \\ communication, Jean-Philippe Durand décrypte pour nous l'offre et l'approche pédagogique \\ de l'un d'entre eux, l'Agence de Gestion des Entreprises Culturelles d'Ille-de-France (AGECIF), \\ dont il est aussi responsable pédagogique et formateur depuis 1991.
}

Pour commencer, pouvez-vous nous dire quelques mots sur la genèse de l'AGECIF ?

L'AGECIF voit le jour en 1984 à l'initiative du ministère de la Culture qui cherche alors à professionnaliser le secteur du spectacle vivant. À l'époque, il existe une forte volonté politique d'affirmer l'importance de la culture dans sa dimension économique. Il faut également se rappeler qu’à cette période le secteur culturel doit composer avec un contexte budgétaire difficile. Pour s'assurer un niveau de ressources suffisant, il lui faut adopter des modalités de gestion aussi rigoureuses que celles du privé. L'AGECIF naît donc dans ce cadre-là, avec au départ des formations qui portent essentiellement sur de la gestion pure, c'est-à-dire le droit ou la comptabilité, pour répondre à des urgences. Par la suite, au fil des années, le périmètre des formations s'élargit à d'autres champs comme le montage de projets, les recherches de financements, la communication, le marketing, et plus récemment, la gestion des ressources humaines ou l'efficacité personnelle et professionnelle. Dans le même temps, l'AGECIF s'ouvre à des domaines comme le patrimoine, les arts plastiques, les musées, le livre et la lecture, avec une idée forte qui est de travailler sur la mixité des publics. Des évolutions interviennent également en matière de publics. Si l'AGECIF s'adresse au départ aux intermittents et aux permanents du monde du spectacle, des dispositifs spécifiques se développent dans les années 1990 avec, par exemple, les programmes pour les emplois jeunes.

Aujourd'hui, comment définiriez-vous les caractéristiques de l'AGECIF par rapport à d'autres organismes en termes de formation?

Dès sa création, l'AGECIF a fait le choix de formations modulaires. Il s'agit de sessions relativement courtes qui peuvent être juxtaposées les unes aux autres, de manière à constituer des parcours complets de formations. L'AGECIF inscrit ses formations dans une logique pluriannuelle. Ainsi, promesse est faite aux participants de reconduire les mêmes thématiques, libre à eux de compléter une année les formations qu'ils n'ont pas réussi à suivre en totalité les années précédentes. Mais, surtout, ce qui distingue l'AGECIF de la plupart des autres centres de formation, et notamment des universités, c'est que chaque module est confié à un responsable pédagogique unique qui en est le seul intervenant, même s'il peut faire appel à des témoins.

\section{Pourquoi un seul intervenant par formation?}

C'est une volonté forte de l'AGECIF. Et elle la revendique fortement. L'AGECIF part du principe que la formation ne peut se limiter à de simples apports de connaissances et de présentations de témoignages. L'AGECIF se positionne, elle, sur des parcours de formations qui intègrent de véritables constructions pédagogiques. Cela signifie aussi que les intervenants ne sont pas forcément des «stars », c'est-àdire qu'un module n'est pas confié à telle ou telle personne parce qu'il a été directeur d'un grand festival par exemple. L'AGECIF choisit plutôt des professionnels qui connaissent bien leur secteur mais qui sont également de véritables pédagogues, formés à la pédagogie, c'est-à-dire capables de raisonner en termes d'acquisitions de compétences, de travailler non pas sur une approche par les contenus mais selon une logique par objectifs, d'identifier le niveau de départ des participants et de choisir les méthodes pour les conduire là où ils souhaitent les emmener.

Au niveau humain, l'AGECIF se définit comme une équipe composée de permanents et d'intervenants. Quel est le profil de toutes ces personnes et comment fonctionnent entre eux tous ces acteurs?

L'équipe compte actuellement treize permanents et une soixantaine de responsables pédagogiques extérieurs. 


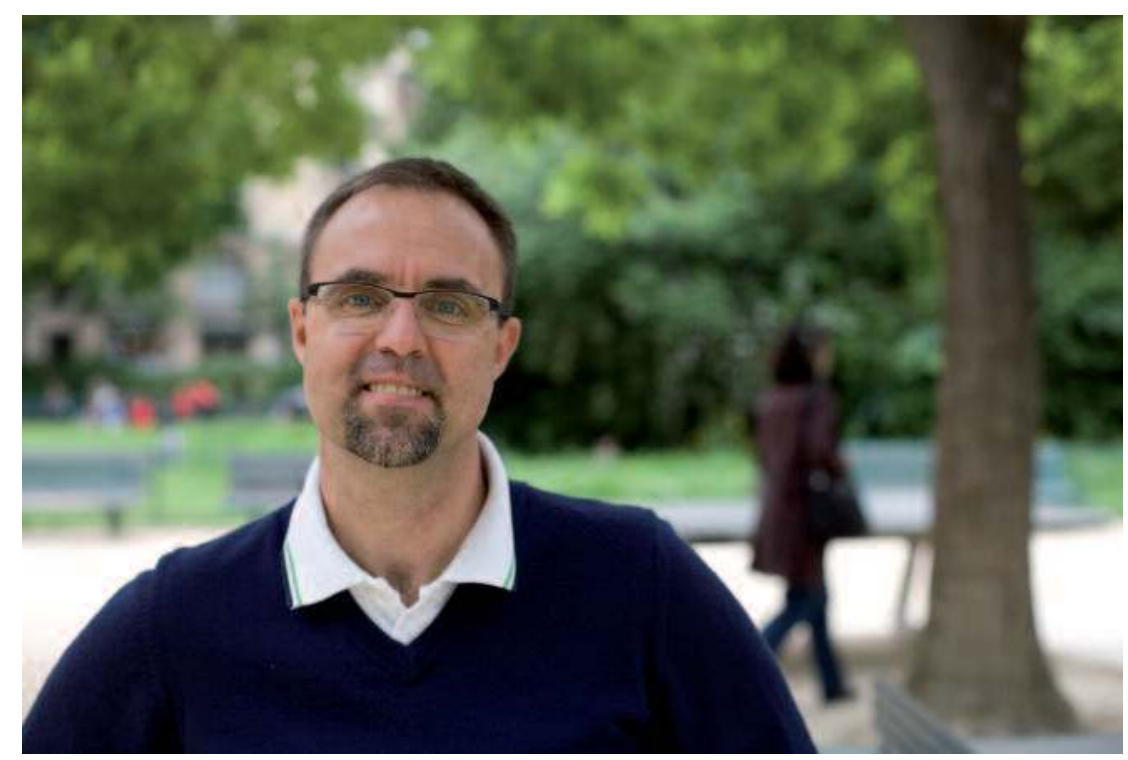

Jean-Philippe Durand, responsable pédagogique à l'AGECIF

Pour choisir ses collaborateurs, l'AGECIF n'a pas de profil type. C'est plutôt le fruit de rencontres, de discussions. En fait, lorsqu'une thématique nouvelle entre au catalogue, les responsables pédagogiques sont les premiers informés, qu'ils se sentent en capacité de l'animer ou qu'ils nous orientent sur une personne en mesure de la prendre en charge. Pour devenir formateur, il faut avant tout une envie de transmettre et, bien sûr, une connaissance spécifique du domaine en tant que professionnel de la culture. C'est ensuite que démarre l'accompagnement en vue d'acquérir les compétences de formateur. Les formations de l'AGECIF abordant une grande diversité de thématiques, notre équipe compte aussi une grande diversité de profils.

Justement, comment se passe l'élaboration de votre catalogue de formations?

L'élaboration d'un catalogue, cela représente 18 mois de réflexion, avec six mois de travail effectif, précédés de douze mois de veille. Le processus comporte plusieurs étapes distinctes. Il y a tout d'abord les idées qui germent un peu naturellement tout au long de l'année et qu'il convient de noter scrupuleusement pour les tester plus tard et en évaluer la faisabilité. Bien sûr, cela nécessite une très bonne connaissance du secteur. Tout cela se double d'une importante veille quotidienne sur l'actualité dans tout un tas de domaines : le droit, le social, la fiscalité, ou encore l'économie, la communication, la sociologie, les nouvelles technologies... Ce travail a pour but de collecter des éléments qui aident à l'analyse des besoins. D'autre part, les évaluations des formations constituent un gisement de données très riche. Elles procèdent par des questionnaires écrits remplis par les participants à l'issue de chaque formation suivis d'un échange à l'oral. Elles prennent aussi la forme d'entretiens réguliers avec les responsables pédagogiques. Par ailleurs, des rendez-vous systématiques sont planifiés avec des responsables d'institutions culturelles, qu'il s'agisse de responsables des ressources humaines ou de responsables opérationnels. Enfin, le contenu des appels d'offres publics complète nos sources d'informations. Toutes ces remontées d'informations nous permettent ensuite d'identifier des besoins qui ne seraient pas encore couverts et de réfléchir aux suites éventuelles à leur donner.

\section{Comment évolue votre offre de formations d'une année à l'autre?}

La plupart des modules sont conservés, d'autres évoluent, certains sont supprimés, mais relativement peu, et, enfin, de nouveaux voient le jour. Le premier travail consiste à faire évoluer les modules en fonction des résultats d'évaluation. Ces changements peuvent porter à la fois sur les contenus, sur les formats, sur les rythmes, sur les méthodes. En ce qui concerne les créations, la plupart sont des succès. Mais nous connaissons aussi des échecs retentissants... Cela tient souvent au fait qu'une attente a été identifiée top tôt, avant de devenir un besoin formalisé de formation. Du coup, l'AGECIF se place plutôt dans une logique d'anticipation d'un besoin. Ainsi, il nous arrive de faire figurer une formation au catalogue qui ne rencontre pas de succès l'année en cours mais trouvera son public deux ou trois ans plus tard. Autre cas de figure, celui de salariés qui évoluent sur un poste où il existe un véritable besoin de formations, auquel le module répond, mais qui demande un certain temps pour être validé par la direction de la structure. De toute façon, il y a toujours une 
prise de risque lorsqu'une nouvelle formation est ajoutée au catalogue. Rien de permet de savoir si elle va fonctionner, même si les problématiques semblent, de prime abord, pertinentes en regard du contexte du moment et si une demande a été identifiée.

\section{Quelles nouveautés figurent au programme de votre offre 2010-2011?}

Pour la rentrée 2010-2011, le catalogue de l'AGECIF intègre trois nouvelles familles : des «modules experts » qui traitent d'une thématique pointue en un temps très court et s'adressent plus particulièrement aux cadres supérieurs et membres des équipes de direction ; des " séminaires », montés essentiellement en partenariat avec l'université Lyon 2 et qui, au-delà de la logique formative, invitent à une ouverture, une prise de recul, sur des problématiques en lien avec l'actualité ; enfin, des formations « English for special purposes », qui se déroulent en anglais et traitent d'une thématique précise. Cette formule permet de renforcer ses capacités linguistiques tout en élargissant ses compétences en matière de diffusion du spectacle, de relations aux publics, d'animation de réunion ou encore d'échanges internationaux. Parallèlement à la création de ces nouvelles familles, l'AGECIF innove en défrichant de nouveaux thèmes : sur la saison 20102011,56 nouvelles formations apparaissent dans un catalogue qui en compte un peu plus de 180.

\section{L'offre de l'AGECIF s'enrichit ainsi chaque année d'un nombre conséquent de nouvelles formations. $\mathrm{Ne}$ craignez-vous pas que cet accroissement continu nuise à la qualité du catalogue?}

Bien au contraire. Et cette richesse explique sans doute le succès des formations et la longévité de l'AGECIF. D'ailleurs, l'AGECIF est repérée comme un lieu d'innovation qui anticipe les besoins en gestion du secteur. Nos publics sont extrêmement fidèles. Et puis si l'AGECIF propose autant de nouvelles formations chaque année, c'est aussi parce qu'elle touche des publics de tous horizons culturels et aux motivations très diverses. Ainsi, des personnes sont intéressées plutôt par les thématiques liées à la communication, d'autres par la gestion... La volonté de chacun est aussi de se spécialiser par rapport à son domaine d'activités principal. Certains stagiaires viennent se former chaque année depuis dix ans. Ce n'est d'ailleurs pas toujours dans une recherche de pur apport en contenu, mais aussi parce que cela leur permet aussi de faire le point en fin de saison, bref, de souffler, d'échanger sur leurs pratiques, de les valider ou de les remettre en cause, de les questionner, de les renforcer ou d'en explorer de nouvelles.
Vous développez aussi des formations sur mesure. De quoi s'agit-il et quelles en sont les principales difficultés?

À côté des formations inter entreprises, l'AGECIF est connue pour développer toute une gamme de formations en intra, qualifiées aussi de «formations sur mesure ». Ici, la difficulté principale est de bien analyser les différentes composantes de la demande et notamment de distinguer ce qui relève d'une attente des personnels et d'une demande de la direction. Ce type de formations fonctionne bien car elles collent au plus près des spécificités de chaque établissement. D'ailleurs une personne qui suit une formation en inter est notre meilleur prescripteur pour organiser une formation en intra. Pour l'AGECIF, il est clair que ce type d'offre est très riche car il permet vraiment d'être au cœur des préoccupations et des besoins d'une structure.

D'un autre côté, ne pensez-vous pas que ces formations sur mesure privent leurs participants d'une certaine dynamique de groupe ou tout du moins d'un échange d'expérience?

Il est bien évident que l'aspect d'ouverture sur l'autre peut sembler moins évident dans ce type de formations. Cela dit, cet effet est contrebalancé par deux qualités importantes. D'une part, la fabrication de contenus très finement articulés à l'expérience professionnelle des participants. D'autre part, la prise de recul des stagiaires de façon collective à partir de grilles communes. Ces formations aident à forger et partager un langage commun. Enfin, et cet aspect n'est pas à négliger, les formations en intra favorisent les échanges non pas avec l'extérieur mais en interne, dans un cadre propice, autorisé et protégé. Car il est des choses qui ne se disent pas naturellement en interne. Soit par manque de temps, soit en raison d'horaires décalés, soit parce que les niveaux hiérarchiques limitent la prise de parole.

Pour déployer vos formations sur l'ensemble du territoire, vous vous appuyez sur une logique de partenariats. Expliquez-nous concrètement comment celle-ci fonctionne.

Le travail en partenariat est une caractéristique très forte à l'AGECIF. Deux types de partenariat ont été élaborés. D'une part, des partenariats nationaux avec des acteurs qui maîtrisent des contenus extrêmement pointus : "Relais Culture Europe », pour des formations sur le montage et le financement de projets européens ; « Hors les Murs », avec qui sont développées des formations spécifiques dans le domaine des arts de la rue et des arts de la piste ; et enfin "Artek », dans le domaine de la prévention des risques et de la sécurité. D'une part, l'AGECIF 
Quel état des lieux peut-on dresser aujourd'hui de l'offre de formation professionnelle continue, diplômante ou non, dans le secteur culturel ? De prime abord, il apparaît difficile de proposer une évaluation purement quantitative pour aborder cette problématique. En effet, il semble compliqué d'établir un recensement exhaustif de tous les organismes (publics ou privés) proposant ce type de service, qui plus est dans un champ extrêmement hétérogène et donc difficile à délimiter. Pour traiter la question de la formation continue dans le champ culturel, il semble donc nécessaire de devoir resserrer la question autour d'un angle précis.

Ainsi, en 2008, le secteur formation de l'OCIM, alors désireux d'actualiser ses connaissances sur son contexte de travail pour tracer de nouvelles perspectives, s'est livré à un début d'analyse autour des principaux organismes dont l'offre « s'approchait » de la sienne. Dans un premier temps, une étude a donc été menée à partir d'Internet pour identifier ces organismes et élaborer par la suite une grille d'analyse commune. Celle-ci comportait l'identification des thèmes des formations, la typologie des formations, les lieux des formations, le nombre des formations, les publics touchés et l'équipe de formateurs. L'analyse a été volontairement ouverte à des domaines extérieurs au champ muséal comme, par exemple, le spectacle vivant, les théâtres, les bibliothèques... Bien sûr, ce travail mériterait d'être poursuivi et approfondi, mais à titre informatif, voici les principaux éléments d'observations qui en ressortent :

- sur les 17 organismes identifiés (CAGEC, AGECIF, ARSEC, AVEC, Premier'Act, OGACA, INP, CNFPT, INET, ENACT, ENSSIB, ADBS, Arcade, CCL, CIPAC, FEMS, SMQ), la gestion et la législation font partie des thèmes des formations proposées par la majorité des structures, suivies par les thèmes de la communication, de la médiation et des publics. Les formations concernant la documentation sont proposées par environ la moitié des organismes identifiés pendant que la conservation et l'exposition sont des sujets développés de façon moins importante. À noter aussi que d'autres thèmes, ne relevant pas de la thématique habituelle de l'OCIM et qui sont proposés, concernent : le management, les archives, les ressources humaines, l'administration, la diffusion ; - concernant le type et les actions de formation, la majorité des organismes proposent des stages d'une durée allant d'une journée à 4 jours. On remarque également une offre sous forme de cycles de formation (AGECIF, Premier'Act, ENACT) où les participants sont invités à suivre plusieurs sessions sur une même thématique. On observe aussi un développement des formations ciblées sur un territoire (par exemple l'IRPA pour la région Bretagne). Les terminologies les plus souvent utilisées sont : stages, formations, colloques...

- la visibilité du contenu proposé : un nombre très faible d'organismes mettent en ligne un contenu détaillé de la formation comportant la liste des intervenants. En fait, le programme détaillé est souvent remplacé par une fiche descriptive avec les objectifs et méthodes pédagogiques proposées, parfois un petit descriptif de la formation, les destinataires auxquels elle s'adresse ou encore le coût ; - pour valoriser leurs actions, les organismes proposent plutôt des pages thématiques, avec l'énumération de l'ensemble des sujets développés. On remarque aussi la présence sur certains sites d'organismes de proposition de parcours, de fiches métier et de compétences pour certaines professions ;

- enfin, on observe que près de la moitié des organismes étudiés proposent des formations à la carte.

La rédaction de la Lettre de l'OCIM, d'après une étude du secteur Formation de l'OCIM s'est associée avec des structures enracinées localement ou régionalement sur un territoire. Avec elles, l'AGECIF « décentralise » certaines formations de son catalogue. Ensuite, à partir des spécificités professionnelles de chacune, sont fabriquées en commun de nouvelles formations qui se dérouleront soit en région, soit à Paris. La saison 2010-2011 est marquée par l'arrivée de deux nouveaux partenaires : "Multicité », une structure basée à Lille et œuvrant dans le domaine du développement local ; "OGACA », un organisme situé à Strasbourg et créé dans la même logique que l'AGECIF au début des années 1980.

\section{Que représente l'offre des formations à destination des professionnels de musées par rapport à l'offre globale? Quelles sont les thématiques concernées?}

De très nombreuses thématiques, présentes au catalogue de l'AGECIF, concernent directement le champ particulier des musées : formations sur la communication, les publics, le développement personnel, les ressources humaines, l'animation et la gestion d'équipes, le Web 2.0 ou encore les droits d'auteurs. À la demande de partenaires, il arrive que l'AGECIF crée des formations très pointues sur tel ou tel secteur d'activités, mais notre 
volonté est plutôt d'élargir le champ de réflexion des participants en les confrontant à ce qui se passe ailleurs. Ainsi, un musée a tout intérêt à analyser les pratiques d'une scène nationale en matière de spectacle vivant. De même, un centre d'art contemporain s'enrichit en étudiant ce qui fonctionne dans le service éducatif d'un musée d'archéologie, qui lui-même gagne à se nourrir de ce qui se passe dans une compagnie théâtrale. C'est vraiment cette idée d'échanges de compétences qui nous intéresse à l'AGECIF. À une même session de formation peuvent participer un musée, un théâtre national, un cinéma d'art et d'essai, un centre d'art, une compagnie théâtrale, un festival. Le fait de travailler de cette façon-là permet aux participants de croiser les regards, interroger leurs propres pratiques, élargir leur champ d'expérimentation. En revanche, l'AGECIF ne développe pas de formations spécifiques sur la conservation préventive. Ce n'est pas son savoir-faire. Alors, si nous étions amenés à créer au niveau national des formations sur ce genre de thématique, il conviendrait de monter un partenariat avec une structure dont c’est la spécialité... comme l'OCIM, par exemple.

Aujourd'hui, qui compose l'essentiel de votre public ? La physionomie de ce public a-t-elle changé dans le temps ?

Globalement, le public des formations de l'AGECIF se compose à plus de $80 \%$ d'intermittents ou de personnes en poste dans des structures, qu'il s'agisse d'associations, d'établissements publics, de services municipaux... Ensuite arrivent les personnes qui effectuent une reconversion professionnelle, et enfin des demandeurs d'emploi mais cette fois dans une moindre proportion, inférieure à $5 \%$. Sinon, la physionomie de ce public a peu évolué au cours des ans. Il y a quand même un trait caractéristique : plus de $80 \%$ sont des femmes. Cela dit, cette statistique est vraie de tous les centres de formations. Il faut aussi avouer que ce phénomène est lié au fonctionnement du secteur culturel qui, statistiquement, compte plus de femmes que d'hommes. Par contre, ce qui a évolué, c'est que les personnes qui y travaillent sont de plus en plus diplômées.

\section{Du point de vue des participants, le rapport à la} formation n'a-t-il tout de même pas un peu changé ?

La demande tend, sans doute, à devenir un peu plus individualiste. Chaque personne veut qu'on s'occupe d'elle et presque uniquement d'elle. À l'AGECIF, notre réponse est de faire travailler les participants ensemble. Pour nous, la formation c'est l'ouverture à l'autre, l'enrichissement réciproque C'est aussi comprendre ce qui se passe ailleurs pour pouvoir transposer une expérience dans sa propre sphère professionnelle. Et pour cela, il faut être à l'écoute. Voilà pourquoi durant les formations nous nous efforçons de faire travailler les personnes ensemble, par exemple par petits groupes sur des études de cas.

\section{Aujourd'hui, quelles sont les motivations principales des personnes qui vous sollicitent?}

Tout dépend de l'âge des personnes et de l'expérience qui est la leur au sein de leur structure. Partant de là, les demandes peuvent être très techniques, sur des points que ces personnes ne maîtrisent pas, dans le domaine de la gestion sociale, par exemple, ou en matière de bureautique ou de langue. Beaucoup de participants souhaitent également opérer une prise de recul par rapport à leur pratique professionnelle. La formation leur donne l'opportunité de valider ou d'invalider des modalités de travail, d'aller plus loin, de se conforter à autrui. En fait, les personnes qui entrent en formation font le constat, à un moment donné dans leurs structures, de leur isolement. Et ce, même si elles travaillent en équipe. Enfin, certaines personnes opèrent une évolution professionnelle et donc, à partir de là, il leur vient l'idée de renforcer leurs compétences actuelles ou d'en acquérir de nouvelles.

\section{Concrètement, pouvez-nous nous décrire les modalités de déroulement d'une formation?}

Il n'y a pas deux formations qui se ressemblent puisqu'elles fonctionnent à partir d'une logique de compétences. Le choix des méthodes pédagogiques employées et leur articulation au sein d'une séquence pédagogique peuvent fortement varier : apports de connaissances sous forme d'exposés, de commentaire de fiches techniques, d'analyse de texte ; exercices pratiques, études de cas, simulations ; partages d'expériences, retours sur expérience, élaboration de cahiers de bonnes pratiques... Toutes ces approches sont extrêmement dynamiques, voire ludiques dans certains cas, et toujours en phase avec l'expérience professionnelle des participants.

\section{Pour vous, qu'est-ce qui fait la qualité d'une formation ?}

La qualité d'une formation est le fruit d'une fragile alchimie. Les compétences pédagogiques du formateur, son implication dans le terrain professionnel et ses capacités d'animateur sont un facteur de poids. Mais pas seulement. La nature et le degré d'implication des participants jouent un rôle majeur. S'y ajoute la qualité de l'accueil.

Selon vous, quelles sont les tendances à venir en matière de formation continue dans le domaine culturel ? En clair, quels sont les grands thèmes porteurs?

Regardez le catalogue 2010-201 l et vous constaterez qu'il s'est fortement étoffé dans le domaine du management 
des personnes, c'est-à-dire tout ce qui porte sur la gestion prévisionnelle des emplois et des compétences, le pilotage et l'animation d'équipe, le fonctionnement en mode projet. Les années 2000 ont été plutôt marquées par l'irruption du Web dans les outils de communication. Et si l'on remonte plus en arrière, les années 1990 ont, quant à elles, surtout été centrées sur la thématique de la médiation.

Le contexte de crise économique a-t-il une influence sur l'offre de l'AGECIF ?

Au niveau des publics de l'AGECIF, le constat est très clair : quand le secteur culturel va mal, les salariés vont mal. L'AGECIF doit donc répondre à des niveaux d'angoisse forcément plus importants en période crise. La qualité de l'accompagnement est alors d'autant plus importante. De leurs côtés, les contenus de formation ne peuvent pas faire abstraction du contexte économique ou social dans la mesure où la crise touche les publics qui fréquentent les équipements culturels. À titre d'exemple, l'AGECIF vient de créer une formation sur la gestion des tensions dans la relation d'accueil. Cette problématique est apparue récemment sans doute en raison d'une morosité ambiante ou d'une difficulté de relation latente dans la population qui impacte en particulier le travail des personnels qui sont à leur contact.
La question de la gestion et du marketing appliqués au domaine particulier de la culture est un sujet impliquant de nombreux questionnements et controverses. En tant que formateur, comment abordez-vous la question de l'intégration de concepts et outils issus du monde de l'entreprise dans les pratiques professionnelles du champ culturel ?

Ne perdons pas de vue que nous ne nous situons ni sur les mêmes échelles, ni sur les mêmes enjeux. Mon point de vue est que ces outils ne peuvent être adaptés sans une solide réflexion préalable sur les valeurs qui fondent la légitimité de la structure, de son projet et de ses missions. Ces concepts doivent donc rester des outils à leur service. En aucune façon ils ne doivent devenir une fin en soi. Je pense vraiment que l'AGECIF n'aurait pas eu le succès qui est le sien si cette question des valeurs partagées avec les participants n'étaient pas complètement prégnante. Encore une fois, c'est cette philosophie qui sert de fil directeur au développement de nos activités.

Entretien réalisé par Olivier Soichot 